\title{
Exposure to breast milk in infancy and risk of breast cancer
}

\author{
Lauren A. Wise ${ }^{1}$, Linda Titus-Ernstoff ${ }^{2}$, Polly A. Newcomb ${ }^{3,4}$, Amy Trentham-Dietz ${ }^{3,5}$, \\ Dimitrios Trichopoulos ${ }^{6}$, John M. Hampton ${ }^{3}$, and Kathleen M. Egan ${ }^{7}$ \\ ${ }^{1}$ Slone Epidemiology Center, Boston University, 1010 Commonwealth Ave, Boston, MA 02215, \\ USA, tel. 617-734-6006, fax. 617-738-5119 \\ 2Dartmouth Medical School, Norris Cotton Cancer Center and Hood Center for Children and \\ Families, One Medical Center Drive, Lebanon NH 03756 USA \\ ${ }^{3}$ Paul P. Carbone Comprehensive Cancer Center, University of Wisconsin, 610 Walnut Street, \\ Madison, WI 53726, USA \\ ${ }^{4}$ Cancer Prevention Program, Fred Hutchinson Cancer Research Center, 1100 Fairview Avenue \\ North, Seattle, WA 98104, USA \\ ${ }^{5}$ Department of Population Health Sciences, University of Wisconsin-Madison, 610 Walnut Street, \\ Madison, WI 53726, USA \\ ${ }^{6}$ Department of Epidemiology, Harvard School of Public Health, 677 Huntington Ave, Boston, MA \\ 02115, USA \\ ${ }^{7} \mathrm{H}$. Lee Moffitt Cancer Center and Research Institute, Medical Research Center, 2nd Floor, \\ 12902 Magnolia Drive, Tampa, FL 33612, USA
}

\begin{abstract}
Early life exposures, such as being breastfed in infancy, may influence the risk of breast cancer in adulthood. We evaluated the risk of breast cancer in relation to ever having been breastfed in infancy among 9,442 women who participated in a population-based, case-control study. Cases were identified through cancer registries in three states (Massachusetts, New Hampshire, and Wisconsin); controls were identified through statewide drivers' license lists or Medicare lists. Data on known and suspected risk factors were obtained through telephone interview. We used unconditional logistic regression to assess the relation of breast cancer with ever having been breastfed and duration of breastfeeding (available for only 19\% of breastfed women) in premenopausal women (1,986 cases and 1,760 controls) and postmenopausal women (2,600 cases and 2,493 controls). We found no evidence that ever having been breastfed in infancy was associated with breast cancer risk in either premenopausal women (odds ratio [OR] $=0.96 ; 95 \%$ confidence interval $[\mathrm{CI}]=0.83-1.10)$ or postmenopausal women $(\mathrm{OR}=0.98 ; 95 \% \mathrm{CI}=0.87-1.10)$. The association did not differ according to breast cancer stage, mother's history of breast cancer, or any other reproductive factor assessed. Likewise, we found no association between breastfeeding duration and risk of breast cancer. Our results do not support the hypothesis that exposure to breast milk in infancy influences the risk of adult breast cancer.
\end{abstract}

\section{Keywords}

Breast cancer; Breastfeeding; Menopausal status; Risk factors

Correspondence to: Lauren A. Wise.

Conflicts of interest: none. 


\section{INTRODUCTION}

Being breastfed in infancy has been hypothesized to influence risk of adult breast cancer [17] via mechanisms including transmission of a mammary tumor virus through lactation [1$8]$, ingestion of excreted organochlorines [9,10], or consumption of growth factors [11]. Some [12-15] but not all $[8,16-18]$ case-control studies suggest a protective effect of being breastfed on breast cancer risk, although the results were statistically significant in only two studies $[12,13]$. Two recent prospective studies showed no overall association [19, 20]; however, a recent meta-analysis suggests that being breastfed in infancy is associated with a significantly reduced risk of premenopausal breast cancer [20]. In the present populationbased case-control study, we evaluated the relation of breast cancer risk with ever having been breastfed and duration of breastfeeding in infancy among women aged 20-74 years. To our knowledge, the analysis includes the largest number of premenopausal women to date among studies on this topic.

\section{MATERIALS AND METHODS}

All female residents of Massachusetts (excluding metropolitan Boston), New Hampshire, and Wisconsin with a new diagnosis of invasive breast cancer (ICD-O version 2 C50.0C50.9) or breast cancer in situ (ICD-O version 2 C50.0-C50.9) reported to each state's cancer registry were eligible for this study. We applied separate age restrictions to invasive (aged 20-69 years) and in situ (aged 20-74 years) cases. According to a protocol approved by the institutional review board of the participating centers, the physician on record for each eligible case subject was contacted by mail to obtain permission to interview the patient. Interviews were conducted from February 1997 to May 2001. Eligibility was limited to case subjects with listed telephone numbers, driver's licenses verified by self-report (if less than 65 years of age), and known dates of diagnosis.

\section{Selection of cases}

A total of 8,066 invasive cases and 2,269 in situ cases were eligible for the study. Of the invasive cases, physicians refused contact with 147 (1.8\%), 302 (3.7\%) were deceased, 215 $(2.7 \%)$ could not be located and $973(12.1 \%)$ refused to participate. Of the $6,429(80 \%)$ invasive cases that were interviewed, 8 cases were considered unreliable by the interviewers, leaving 6,421 invasive cases available for analysis. Of the in situ cases, physicians refused contact with $58(2.6 \%), 17(0.7 \%)$ were deceased, 63 (2.8\%) could not be located and 244 $(10.8 \%)$ refused to participate. Of the 1,887 (83\%) in situ cases that were interviewed, 9 cases were considered unreliable by the interviewers, leaving 1,878 in situ cases available for analysis. Of the interviewed invasive and in situ cases ( $\mathrm{N}=8,299)$, more than $98 \%$ were confirmed by histology, cytology, or other means according to the registry reports.

\section{Selection of controls}

Controls were randomly selected in each state from the community using two sampling frames: those under 65 years of age were selected from a list of licensed drivers, and those 65 to 74 years of age were selected from a roster of Medicare beneficiaries compiled by the Centers for Medicare \& Medicaid Services, formerly the Health Care Financing Administration. Controls were selected at random within 5-year age strata to yield an age distribution similar to the cases enrolled in each state. Controls were required to have no personal history of breast cancer, a listed telephone number, and, if less than 65 years of age, a self-reported driver's license. Of the 10,690 potential controls, $86(0.8 \%)$ were deceased, $475(4.4 \%)$ could not be located, and 2,074 (19.4\%) refused to participate. Of the 8,055 $(75 \%)$ controls who were interviewed, 14 were considered unreliable by the interviewers, leaving 8,041 controls available for analysis. 


\section{Data collection}

Case subjects and controls were sent letters briefly describing the study before they were contacted by telephone by trained interviewers. The 40-minute structured telelephone interview elicited data on demographic, lifestyle and behavioral factors, height and weight, reproductive and medical history, and hormone use. Menopause was defined as the absence of periods for $\geq 6$ months before the reference date. Women who reported taking hormones and still having periods, and women who reported hysterectomy alone were classified as premenopausal if their reference ages were in the lowest decile of age at natural menopause among controls, as postmenopausal if their reference ages were in the highest decile, or as unknown menopausal status if their reference ages were between the lowest and highest deciles. Beginning in the second year of the study, women were asked: "Were you breastfed as a baby?" (response categories: yes, no, don't know). To gather additional information on breastfeeding, approximately midway through the study enrollment period, we also asked women who reported being breastfed in infancy to report the duration of breast feeding (months). For $91 \%$ of invasive cases, $95 \%$ of in situ cases, and $94 \%$ of controls, the interviewers reported being unaware of the woman's case-control status until the end of the interview. The reference date was the registry-supplied date of diagnosis for cases. For comparability, controls were assigned a reference date approximately one year before the interview.

\section{Reliability substudy}

To assess the reliability of responses to the questionnaire, a sequential sample of cases and controls from Wisconsin and New Hampshire was re-interviewed. Approximately $88 \%$ of cases and $85 \%$ of controls agreed to be contacted for a second interview. After an average of 3 months (range: $1-5$ months), $98 \%$ of cases $(\mathrm{N}=135)$ and $95 \%$ of controls $(\mathrm{N}=159)$ were successfully recontacted and re-interviewed. Cohen's kappas for the agreement in reports breastfeeding status $(\mathrm{N}=201)$ were 0.88 for controls and 0.96 for cases, and were $0.96,0.86$, and 0.91 for women aged $<48,48-54$, and $55+$ at interview, respectively, suggesting adequate recall of breastfeeding status. We did not collect reliability data on breastfeeding duration.

\section{Exclusions}

Of the 8,299 cases and 8,041 controls interviewed, we excluded 2,173 cases and 2,374 controls who were not asked about breastfeeding, 1,199 cases and 1,104 controls who did not know their breastfeeding status, and 16 cases and 32 controls with missing data on age, parity, or age at first birth, leaving 4,911 cases (3,779 invasive and 1,132 in situ) and 4,531 controls (4,433 for invasive cases and 4,531 for in situ cases) for analysis. No material differences were found between women who were and were not asked about their breastfeeding status. Among women who received a version of the questionnaire that asked about breastfeeding status, the proportion who reported "don't know" was equally distributed among cases and controls (19.5\% vs. 19.5\%). Although these women tended to be older and less educated than women who reported their breastfeeding status (age: 56.0 vs. 53.8 years; college-educated: $23.1 \%$ vs. $28.8 \%$ ), they were similar with respect to other breast cancer risk factors including age at menarche ( 12.8 vs. 12.7 years), parity ( 2.5 vs. 2.5 births), age at menopause ( 48.5 vs. 48.2 years), ever use of female hormones (39.5\% vs. $35.8 \%$ ), recent use of mammography ( $85.6 \%$ vs. $85.3 \%$ ), and family history of breast cancer $(16.0 \%$ vs. $17.0 \%)$. A larger proportion of women from Wisconsin did not know their breastfeeding status relative to Massachusetts and New Hampshire $(23.2 \%, 14.4 \%$, and $15.7 \%$, respectively).

Among the 3,900 women who answered positively to ever having been breastfed, 1,899 $(48.7 \%)$ were asked about duration of breastfeeding. Only 737 (38.8\%) of these women 
were able to provide data on breastfeeding duration, leaving complete data on breastfeeding duration for $19.0 \%$ of breastfed women. Those who were not able to report data on breastfeeding duration tended to be less educated (college-educated: $25.7 \%$ vs. $32.0 \%$ ), but were similar to those with complete duration data with respect to study outcome (case: 51.9 vs. $48.6 \%$ ) and breast cancer risk factors (age: 57.8 vs. 56.0 years; age at menarche: 12.8 vs. 12.7 years; parity: 2.8 vs. 2.7 births; age at menopause: 48.9 vs. 48.7 years; ever use of female hormones: $42.7 \%$ vs. $39.8 \%$, recent use of mammography: $88.3 \%$ vs. $88.9 \%$, and family history of breast cancer: $19.4 \%$ vs. $17.4 \%$ ).

Data Analysis-We used multivariable logistic regression to estimate odds ratios (OR) and $95 \%$ confidence intervals (CI) for breast cancer in relation to ever having been breastfed and breastfeeding duration (available for only $19 \%$ of breastfed women). We controlled for known or suspected confounders, including reference age (years), state of residence (MA, WI, NH), education ( $<$ high school diploma, high school diploma, some college, $\geq$ college degree), Jewish ethnicity (yes, no), body mass index $\left(<20,20-24,25-29, \geq 30 \mathrm{~kg} / \mathrm{m}^{2}\right)$, age at menarche $(<12,12,13,14, \geq 15$ years $)$, parity $(0,1,2,3, \geq 4)$, age at first birth $(<20,20-24$, $25-29, \geq 30$ years), family history of breast cancer (no, yes, unknown), menopausal status (premenopausal, postmenopausal, unknown), age at menopause $(<45,45-49,50-54, \geq 55$ years), and use of postmenopausal hormones (current, past, never). Missing covariate data were modeled using indicator variables. Because similar associations were obtained in ageadjusted and multivariable models, we present results from multivariable models only.

We stratified our analyses by potential effect modifiers of interest such as parity, menopausal status, age at menopause, use of postmenopausal hormones, and mother's history of breast cancer. Birth year (<1946 vs. $\geq 1946)$ was also a stratification variable of interest because the organochlorine DDT was introduced into the U.S. food chain in 1946 [21]. We formally tested for interaction using the likelihood ratio test comparing models with and without cross-product terms between breastfeeding status and these selected factors. As a comparison to results based on the reported breastfeeding duration data (available for $19 \%$ of breastfed women), we used multiple imputation to impute missing breastfeeding duration values dependent on the participant's measured characteristics [22]. With this method, we assumed that the data were missing at random dependent on the participant's characteristics. All p-values were two-sided at the 0.05 level of significance. Analyses were conducted using SAS version 9.2 (SAS Institute, Cary, NC).

\section{RESULTS}

Reproductive factors, such as age at menarche and parity, and family history of breast cancer were associated with breast cancer in the expected direction (Table 1). Overall, 1,843 $(40.7 \%)$ controls and 2,057 (41.9\%) cases reported being breastfed in infancy (Table 2). The median age of women interviewed about their breastfeeding status was 55 years (interquartile range: 48-62 years). In the combined case group, the multivariable OR for breast cancer associated with being breastfed was 0.98 (95\% CI=0.90-1.08). Findings for invasive breast cancer cases were similar to those for the combined case group.

Multivariable ORs were uniform across categories of parity status, menopausal status, age at menopause, postmenopausal hormone use, and mother's history of breast cancer.

Multivariable ORs also did not vary according to year of birth (before or after 1946).

Among the $19 \%$ of breastfed women who reported data on breastfeeding duration, there was digit preference at $1(28 \%), 2(14 \%), 6(19 \%)$, and 9 (11\%) months (data not shown). While the duration category of 3-6 months was inversely related to risk of breast cancer among all cases $(\mathrm{OR}=0.72,95 \% \mathrm{CI}=0.55-0.93)$, the $\mathrm{OR}$ was attenuated when we confined the case group to invasive breast cancers $(\mathrm{OR}=0.77,95 \% \mathrm{CI}=0.58-1.02)$ and there was no evidence 
of a dose-response relation (Table 3). When we imputed the values for breastfeeding duration using multiple imputation (data not shown), the ORs among all cases were 0.97 (95\% CI=0.79-1.18), 0.98 (95\% CI=0.87-1.10), and 1.06 (95\% CI=0.85-1.32) for <3, 3-6, and $>6$ months, respectively, compared with non-breastfed women.

\section{DISCUSSION}

In this large population-based case-control study, we found no evidence of an association between ever having been breastfed in infancy and breast cancer risk in adult women [23]. Findings were null regardless of menopausal status and other evaluated reproductive factors. Moreover, our findings do not lend support to the hypothesis that infant exposure to breast milk containing DDT, introduced to the food chain in the US in the mid-1940s, increases breast cancer risk in adult women. Only a small proportion of women in our study (27\%) were breastfed after the mid-1940s, but DDT exposure was virtually ubiquitous, so nearly all breastfed children of that era would have ingested DDT and its metabolites. Finally, there was limited evidence of an association between duration of breastfeeding and risk of breast cancer, although duration data were only available for $19 \%$ of breastfed women.

Our results for ever having been breastfed are consistent with many, though not all, published studies on this topic. Two case-control studies have shown a modest (26\%) statistically significant reduced risk overall [12] or in young women ( $<45$ years)[13]; however, both studies had low participation rates. A third case-control study found a small (14\%) statistically non-significant reduced risk [14], and an early case-control study based on small numbers found no association [16]. A Swedish nested case-control study with record linkage also produced null findings, but there was limited variation in breastfeeding prevalence (98\%) [17]. The prospective Nurses' Health Study found no association [19], as did a smaller prospective study, the Boyd Orr cohort [20].

Three studies evaluated risk of ever having been breastfed separately in premenopausal and postmenopausal women $[12,15,19]$ and another was limited to premenopausal women only [18], but none found a statistically significant association among premenopausal women. One of the previous reports, based on an earlier phase of this study and an entirely different group of participants, suggested a 35\% reduced risk among premenopausal women [15]. However, the analysis relied on 205 premenopausal cases all of whom were of age 50 years or older; thus, statistical power was limited and the sample did not represent the age spectrum of most premenopausal cases. A recent meta-analysis [20] of studies published prior to 2006 [12-20] (including our earlier study [15]) reported a possible inverse association limited to premenopausal women. The present study, which enrolled a broad age range of participants (20-74 years) and large numbers of premenopausal cases $(\mathrm{N}=1,986)$, would have attenuated the summary estimate if included in the meta-analysis [20].

Study strengths include a large sample size and the use of structured telephone interviews to collect detailed information on a wide range of potential confounders. To our knowledge, the present study included a far larger number of premenopausal cases than in any previous study on this topic. Statistical power was therefore sufficient to detect moderate associations among premenopausal and postmenopausal women. Moreover, the era of the study (birth years 1922-1976) witnessed great variation in the prevalence of breastfeeding as well as the introduction of organochlorines and related breast milk contaminants [23]. Our data are consistent with trends in the prevalence of breastfeeding in the United States, showing higher rates of breastfeeding before the 1950s and steady declines until the early 1980s [23, 24]. 
Study limitations include the inability to validate breastfeeding reports and insufficient data on breastfeeding duration. Because we analyzed breastfeeding status as a dichotomous exposure variable, we cannot rule out the possibility out that our null findings are explained by non-differential (random) misclassification. While we did not validate women's recall of breastfeeding status with reports from her mother (as was done in a previous null study [19]), we found high reliability in the report of breastfeeding status across two questionnaires completed by the same participant.

Due to the retrospective nature of data collection, recall bias may have influenced our findings; however, no differences were found in breast cancer risk factors comparing women who were and were not able to report their breastfeeding status. Moreover, it is unlikely that the exclusion of women with unknown breastfeeding status would have resulted in selection bias because these women were equally distributed among cases and controls. Likewise, the breastfed women who were unable to recall duration did not differ appreciably from the women who could recall with respect to breast cancer risk factors. We found null results for breastfeeding duration and risk of breast cancer using both complete data and multiple imputation techniques.[22] Nonetheless, caution should be used when interpreting our findings on breastfeeding duration due to the small proportion of breastfed women with complete data on duration (19\%).

The present study adds to the growing literature regarding exposure to breast milk in infancy and breast cancer risk in adulthood. Consistent with earlier studies on breast cancer, our findings do not support any protection or hazard associated with having been breastfed in infancy. Given that existing studies have had limited exposure data regarding duration and patterns of exposure to breast milk, future studies might explore for how long the breastfeeding extended and whether the participants were exclusively breastfed.

\section{Acknowledgments}

Financial support: This study was supported by National Cancer Institute grants R01 CA47147, R01 CA47305, and R01 CA69664.

The authors are grateful to Drs. Henry Anderson, Patrick L. Remington, Meir J. Stampfer, Walter C. Willett, John A. Baron, and E. Robert Greenberg; Laura Stephenson and the staff of the Wisconsin Cancer Reporting System, Susan T. Gershman and the staff of the Massachusetts Tumor Registry; Marguerite Stevens and the staff of the New Hampshire Cancer Registry; and Linda Haskins, Heidi Judge, Laura Mignone, and Shafika Abrahams-Gessel along with the study interviewers and programmers in all three states for assistance with data collection. We are especially grateful to the study participants, whose generosity made this research possible.

\section{Abbreviations}
OR odds ratio
CI confidence interval

\section{References}

1. Bittner JJ. Some possible effects of nursing on the mammary gland tumor incidence in mice. Science. 1936; 84:162-3. [PubMed: 17793252]

2. Penrose LS, MacKenzie HJ, Karn MN. A genetical study of human mammary cancer. Br J Cancer. 1948; 2:168-76. [PubMed: 18099716]

3. Tokuhata GK. Morbidity and mortality among offspring of breast cancer mothers. Am J Epidemiol. 1969; 89:139-53. [PubMed: 5765954]

4. Wang Y, Holland JF, Bleiweiss IJ, et al. Detection of mammary tumor virus env gene-like sequences in human breast cancer. Cancer Res. 1995; 55:5173-9. [PubMed: 7585568] 
5. Levine PH, Mesa-Tejada R, Keydar I, et al. Increased incidence of mouse mammary tumor virusrelated antigen in Tunisian patients with breast cancer. Int J Cancer. 1984; 33:305-8. [PubMed: 6321360]

6. Ziegler J. An unlikely link? Researchers probe viral role in breast cancer. J Natl Cancer Inst. 1997; 89:608-10. [PubMed: 9150180]

7. McCann J. Infections and cancer: viruses are still prime suspects. J Natl Cancer Inst. 1998; 90:41820. [PubMed: 9521163]

8. Bucalossi P, Veronesi U. Some observations on cancer of the breast in mothers and daughters. $\mathrm{Br} \mathbf{J}$ Cancer. 1957; 11:337-47. [PubMed: 13499783]

9. Jensen, AA.; Slorach, SA. Chemical contaminants in human milk. Bocan, FL: CRC Press; 1991.

10. Darbre PD. Environmental contaminants in milk: the problem of organochlorine xenobiotics. Biochem Soc Trans. 1998; 26:106-12. [PubMed: 9649729]

11. Morriss, FH. Growth factors in milk. In: Howell, RR.; Morriss, FH.; Pickering, LK., editors. Human milk in infant nutrition and health. Springfield, IL: Charles C. Thomas; 1986.

12. Freudenheim JL, Marshall JR, Graham S, et al. Exposure to breastmilk in infancy and the risk of breast cancer. Epidemiology. 1994; 5:324-31. [PubMed: 8038247]

13. Weiss HA, Potischman NA, Brinton LA, et al. Prenatal and perinatal risk factors for breast cancer in young women. Epidemiology. 1997; 8:181-7. [PubMed: 9229211]

14. Brinton LA, Hoover R, Fraumeni JF Jr. Reproductive factors in the aetiology of breast cancer. Br J Cancer. 1983; 47:757-62. [PubMed: 6860545]

15. Titus-Ernstoff L, Egan KM, Newcomb PA, et al. Exposure to breast milk in infancy and adult breast cancer risk. J Natl Cancer Inst. 1998; 90:921-4. [PubMed: 9637142]

16. Henderson BE, Powell D, Rosario I, et al. An epidemiologic study of breast cancer. J Natl Cancer Inst. 1974; 53:609-14. [PubMed: 4369771]

17. Ekbom A, Hsieh CC, Trichopoulos D, et al. Breast-feeding and breast cancer in the offspring. Br $\mathbf{J}$ Cancer. 1993; 67:842-5. [PubMed: 8471443]

18. Sanderson M, Williams MA, Daling JR, et al. Maternal factors and breast cancer risk among young women. Paediatr Perinat Epidemiol. 1998; 12:397-407. [PubMed: 9805713]

19. Michels KB, Trichopoulos D, Rosner BA, et al. Being breastfed in infancy and breast cancer incidence in adult life: results from the two nurses' health studies. Am J Epidemiol. 2001; 153:275-83. [PubMed: 11157415]

20. Martin RM, Middleton N, Gunnell D, Owen CG, Smith GD. Breast-Feeding and Cancer: The Boyd Orr Cohort and a Systematic Review With Meta-Analysis. J Natl Cancer Inst. 2005; 97:1446-57. [PubMed: 16204694]

21. Hill EL, Morlan HB, Utterback BC, Schubert JH. Evaluation of County Wide DDT Dusting Operations in Murine Typhus Control (1946 through 1949). Am J Public Health Nations Health. 1951; 41:396-401. [PubMed: 14819409]

22. Zhou XH, Eckert GJ, Tierney WM. Multiple imputation in public health research. Stat Med. 2001; 20:1541-9. [PubMed: 11343373]

23. Wolf JH. Low breastfeeding rates and public health in the United States. Am J Public Health. 2003; 93:2000-10. [PubMed: 14652321]

24. Weimer, J. ERS Food Assistance and Nutrition Research Report No. 13. Washington, DC: USDA; 2001. The economic benefits of breastfeeding: a review and analysis. 
Table 1

Overall distribution of risk factors according to breast cancer case and control status

\begin{tabular}{|c|c|c|}
\hline Characteristic & No. $(\%)$ of controls $(N=4,531)$ & No. $(\%)$ of cases $(N=4,911)$ \\
\hline \multicolumn{3}{|l|}{ State } \\
\hline Wisconsin & $2,217(48.9)$ & $2,884(58.7)$ \\
\hline Massachusetts & $1,665(36.8)$ & $1,429(29.1)$ \\
\hline New Hampshire & $649(14.3)$ & $598(12.2)$ \\
\hline \multicolumn{3}{|l|}{ Education } \\
\hline$<$ High school diploma & $320(7.1)$ & $293(6.0)$ \\
\hline High school diploma & $1,735(38.3)$ & $1,882(38.3)$ \\
\hline Some college & $1,217(26.9)$ & $1,238(25.2)$ \\
\hline$\geq$ College degree & $1,242(27.4)$ & $1,475(30.0)$ \\
\hline Missing & $17(0.4)$ & $23(0.5)$ \\
\hline \multicolumn{3}{|l|}{ Religion/Ethnicity } \\
\hline Not Jewish & $4,405(97.2)$ & $4,777(97.3)$ \\
\hline Jewish & $77(1.7)$ & $95(1.9)$ \\
\hline Missing & $49(1.1)$ & $39(0.8)$ \\
\hline \multicolumn{3}{|l|}{ Body mass index $\left(\mathrm{kg} / \mathrm{m}^{2}\right)$} \\
\hline$<20$ & $305(6.7)$ & $317(6.5)$ \\
\hline $20-24$ & $1,836(40.5)$ & 2,097 (42.7) \\
\hline $25-29$ & $1,436(31.7)$ & $1,515(30.8)$ \\
\hline$\geq 30$ & $916(20.2)$ & $942(19.2)$ \\
\hline Missing & $38(0.9)$ & $40(0.8)$ \\
\hline \multicolumn{3}{|c|}{ Family history of breast cancer in mother and/or sister } \\
\hline No & $3,870(85.4)$ & $3,783(77.0)$ \\
\hline Yes & $584(12.9)$ & $1,024(20.9)$ \\
\hline Unknown & $77(1.7)$ & $104(2.1)$ \\
\hline \multicolumn{3}{|l|}{ Age at menarche, years } \\
\hline$<12$ & $876(19.3)$ & $1,033(21.0)$ \\
\hline 12 & $1,018(22.5)$ & $1,207(24.6)$ \\
\hline 13 & $1,312(29.0)$ & $1,385(28.2)$ \\
\hline 14 & $707(15.6)$ & $717(14.6)$ \\
\hline$\geq 15$ & $580(12.8)$ & $515(10.5)$ \\
\hline Missing & $38(0.8)$ & $54(1.1)$ \\
\hline \multicolumn{3}{|l|}{ Parity } \\
\hline 0 & $504(11.1)$ & $661(13.4)$ \\
\hline 1 & $505(11.2)$ & $578(11.8)$ \\
\hline 2 & $1,360(30.0)$ & $1,649(33.6)$ \\
\hline 3 & $1,041(23.0)$ & $1,070(21.8)$ \\
\hline$\geq 4$ & $1,121(24.7)$ & $953(19.4)$ \\
\hline \multicolumn{3}{|c|}{ Age at first term pregnancy, years } \\
\hline$<20$ & $786(19.5)$ & $706(16.6)$ \\
\hline
\end{tabular}




\begin{tabular}{|c|c|c|}
\hline Characteristic & No. $(\%)$ of controls $(N=4,531)$ & No. $(\%)$ of cases $(\mathrm{N}=4,911$ \\
\hline $20-24$ & $1,943(48.2)$ & $1,893(44.5)$ \\
\hline $25-29$ & $920(22.9)$ & $1,121(26.4)$ \\
\hline$\geq 30$ & $378(9.4)$ & $530(12.5)$ \\
\hline \multicolumn{3}{|l|}{ Menopausal status } \\
\hline Premenopausal & $1,760(38.8)$ & $1,986(40.4)$ \\
\hline Postmenopausal & $2,493(55.1)$ & $2,600(53.0)$ \\
\hline \multicolumn{3}{|l|}{ Age at menopause: } \\
\hline$<45$ & $499(11.0)$ & $386(7.9)$ \\
\hline $45-49$ & $512(11.3)$ & $542(11.0)$ \\
\hline $50-54$ & $760(16.8)$ & $824(16.8)$ \\
\hline$\geq 55$ & $202(4.5)$ & $303(6.2)$ \\
\hline Unknown/missing & $520(11.5)$ & $545(11.1)$ \\
\hline Unknown & $278(6.1)$ & $325(6.6)$ \\
\hline \multicolumn{3}{|l|}{ Use of menopausal hormones } \\
\hline Never & $2,943(65.0)$ & $3,083(62.8)$ \\
\hline Former & $241(5.3)$ & $262(5.3)$ \\
\hline Current & $1,323(29.2)$ & $1,535(31.3)$ \\
\hline Missing & $24(0.5)$ & $31(0.6)$ \\
\hline \multicolumn{3}{|c|}{ Mammography use (previous 5 yrs) } \\
\hline Never & $637(12.8)$ & $739(15.0)$ \\
\hline $1-2$ & $1,026(22.6)$ & $756(15.4)$ \\
\hline $3-4$ & $551(12.2)$ & $518(10.5)$ \\
\hline 5 & $1,971(43.5)$ & $2,430(49.5)$ \\
\hline$\geq 6$ & $315(7.0)$ & $421(8.6)$ \\
\hline Missing & $41(0.9)$ & $47(1.0)$ \\
\hline
\end{tabular}


